\title{
A SHARP UPPER BOUND FOR DEPARTURE FROM NORMALITY
}

\author{
Steven L. Lee
}

\begin{abstract}
Mathematical Sciences Section
Oak Ridge National Laboratory

P.O. Box 2008, Bldg. 6012

Oak Ridge, TN 37831-6367

slee@msr.epm.ornl.gov
\end{abstract}

Date Published: August 1993

Research was supported by the Applied Mathematical Sciences Research Program of the Office of Energy Research, U.S. Department of Energy.

Prepared by the

Oak Ridge National Lithoratory

Oak Ridge, Tennessee 37831

managed by

Martin Marietta Energy Systems, Inc.

for the

U.S. DEPARTMENT OF ENERGY

under Contract No. DE-AC05-84OR21400 


\section{Contents}

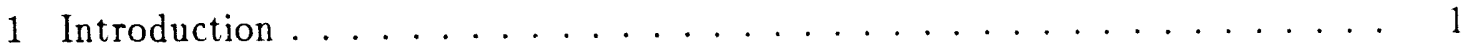

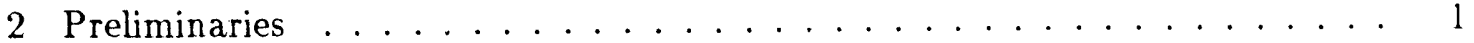

3 A Sharp Upper Bound . . . . . . . . . . . . . . . . . . 5

4 Numerical Results . . . . . . . . . . . . . . . . . . . 9

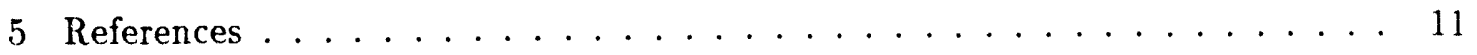




\title{
A SHARP UPPER BOUND FOR DEPARTURE FROM NORMALITY
}

\author{
Steven L. Lee
}

\begin{abstract}
The departure from normality of a matrix is a real scalar that is impractical to compute if a matrix is large and its eigenvalues are unknown. A simple formula is presented for computing an upper bound for departure from normality in the Frobenius norm. This new upper bound is cheaper to compute than the upper bound derived by Henrici [Numer. Math., 4 (1962), pp. 24-40]. Moreover, the new bound is shalp for Hermitian matrices, skew-Hermitian matrices and, in general, any matrix with eigenvalues that are horizontally or vertically aligned in the complex plane. I a terms of applications, the new bound can be used in computing bounds for the spectral norm of matrix functions or bounds for the sensitivity of eigenvalues to matrix perturbations.
\end{abstract}




\section{Introduction}

The departure from normality of a matrix. like the condition number of a matrix, is a real scalar that can be used to compute bounds for various matrix computations. For example, departure from normality can be used to bound the powers, inverses, spectral variation, and fields of values of nonnormal matrices [5] or bound the spectral norm of matrix functions [1]. Unforturately, the departure from normality of a matrix is impractical to compute if a matrix is large and its eigenvalues are unknown. The main result of this paper is a simple formula for computing an upper bound for the departure from normality of a matrix in the Frobenius norm. This new upper bound is cheaper to compute than the upper bound derived by Henrici [5], and it is sharp for any matrix with eigenvalues that are horizontally or vertically aligned in the complex plane. The practical significance is that the new upper bound can be used in computing bounds for many of the matrix computations described in $[1,5]$.

The outline of this paper is as follows. In $\S 2$, we establish notation, motivate the definition of the departure from normality of a matrix, and give Henrici's upper bound for departure from normality [5]. In $§ 3$, we derive a new upper bound for departure from normality and prove that it is sharp for certain classes of matrices. In $\S 4$, we conclude with some numerical results that compare the tightness of Henrici's bound and the new bound.

\section{Preliminaries}

Let $A=\left(a_{i j}\right)$ denote an $n \times n$ complex matrix and let $A^{H}=\left(\bar{a}_{j i}\right)$ denote the conjugate transpose of $A$. (Herein . all matrices are square matrices of order $n$ with real or complex entries.) Several important classes of matrices are defined in terms of their conjugate transpose: for example, $A$ is a Hermitian matrix if and only if (iff) $A^{H}=A, A$ is a skewHermitian matrix iff $A^{H}=-A$, and $A$ is a unitary matrix iff $A^{H} A=A A^{H}=1$. Let $M$ and $N$ denote the Hermitian part of $A$ and the skew-Hermitian part of $A$, respectively. Indeed, let the functions $\mathcal{H}(\cdot)$ and $\mathcal{S}(\cdot)$ be the Hermitian part and skew-Hermitian part of any square matrix. Then, for

$$
\mathcal{H}(A):=\frac{1}{2}\left(A+A^{H}\right) \equiv M
$$

and

$$
\mathcal{S}(A):=\frac{1}{2}\left(A^{H}-A\right) \equiv N,
$$

$A$ has the splitting

$$
A=M-N
$$


Let $R$ denote an upper triangular matrix, $T$ denote a strictly upper triangular matrix, and $\Lambda$ denote a diagonal matrix whose entries are the eigenvalues, $\lambda_{i}$, of $A$. Let $\operatorname{Re}(\Lambda)$ and $\operatorname{Im}(\Lambda)$ denote diagonal matrices whose entries are the real parts and imaginary parts of the eigenvalues of $A$, respectively. Finally, recall that the sum of the eigenvalues of a matrix is equal to the trace of the matrix, where

$$
\operatorname{trace}(A)=\sum_{i=1}^{n} a_{i i} .
$$

The Schur decomposition states that every square matrix $A$ is unitarily similar to an upper triangular matrix $R$.

Theorem 2.1 (Schur decomposition). Given a square matrix $A$, there is a unitary matrix $U$ such that

$$
U^{H} A U=R
$$

The matrix $U$ is not unique and may be chosen so that the eigenvalues of $A$ appear on the diagonal of $R$ in any order.

If $U=\left[u_{1}, \ldots, u_{n}\right]$ is a column partitioning of the unitary matrix $U$ in $(5)$, then the $u_{i}$ are referred to as Schur vectors [4]. For concreteness, let $U$ denote a particular unitary matrix whose columns are Schur vectors so that $R$ is unique and has the splitting

$$
R=\Lambda+T
$$

We now show the connection between the Schur decomposition of $A$ and certain matrix norms of $A$.

Definition 2.2. Let

$$
\|A\|_{F}=\sqrt{\sum_{i, j}\left|a_{i j}\right|^{2}}
$$

and

$$
\|A\|_{2}=\sqrt{\lambda_{\max }\left(A^{H} A\right)}
$$

The Frobenius norm $\|\cdot\|_{F}$ and the spectral norm $\|\cdot\|_{2}$ are invariant under unitary similarity transformations; that is,

$$
\|A\|_{F}=\left\|U^{H} A U\right\|_{F}=\|R\|_{F}
$$

and

$$
\|A\|_{2}=\left\|U^{H} A U\right\|_{2}=\|R\|_{2}
$$


The Frobenius norm and the Schur decomposition play an important role in the definition of normal matrices.

Definition 2.3. [6] The matrix $A$ is normal if and only if any of the following equivalent statements hold:

(a) A has a complete, unitary set of eigenvectors,

(b) $\|A\|_{F}=\|\Lambda\|_{F}$, or

(c) $A^{H} A=A A^{H}$.

Normal matrices include Hermitian matrices, skew-Hermitian matrices, unitary matrices and, in general, any matrix that is unitarily similar to a diagonal matrix. Hence, any Schur decomposition of a normal matrix gives

$$
U^{H} A U=R=\Lambda+T
$$

where $T=0$. For a matrix that is not normal, it is convenient to quantify its departure from normality in terms of a norm of $T$. Any matrix norm can be used; however, the Frobenius norm of $T$ is a natural choice since it is easy to compute and its value is the same for all Schur decompositions.

Definition 2.4 (Departure from Normality). [5] For any $n \times n$ matrix $A$,

$$
\operatorname{dep}_{F}(A):=\|T\|_{F}=\left(\|A\|_{F}^{2}-\|\Lambda\|_{F}^{2}\right)^{1 / 2} .
$$

This definition of departure from normality, $\operatorname{dep}_{F}(A)$, follows from the Schur decomposition of $A$ and equation (9) via

$$
\|A\|_{F}^{2}=\left\|U^{H} A U\right\|_{F}^{2}=\|R\|_{F}^{2}=\|\Lambda+T\|_{F}^{2}=\|\Lambda\|_{F}^{2}+\|T\|_{F}^{2}
$$

so that

$$
\operatorname{dep}_{F}(A):=\|T\|_{F}=\left(\|A\|_{F}^{2}-\|\Lambda\|_{F}^{2}\right)^{1 / 2}
$$

is independent of the choice of $U$. It is also easy to prove that $\operatorname{de}_{F}(A)$ is independent of complex shifts and unitary scalings (i.e., rotations). For example, in the Schur decomposition of $A-\alpha I$, where

$$
U^{H}(A-\alpha I) U=R-\alpha I=\Lambda+T-\alpha I,
$$

note that the shift only alters the diagonal elements of $R$ and that $\operatorname{dep}_{F}(A):=\|T\|_{F}$ is unchanged. Hence,

$$
\operatorname{dep}_{F}(A)=\operatorname{dep}_{F}\left(e^{-i \theta}(A-\alpha I)\right)
$$


for the complex scalar $\alpha$ and $0 \leq \theta<2 \pi$. Later, we show the significance of this observation.

For normal matrices, $\operatorname{dep}_{F}(A)=0$ via Definition $2.3(\mathrm{~b})$. For nonnormal matrices, $\operatorname{dep}_{F}(A)$ is the nonzero quantity defined by (12). To be clear. a small example helps to summarize the main ideas up to this point.

Example 1. Consider two different Schur decompositions of

$$
A=\left(\begin{array}{ccc}
4 & \sqrt{1 / 2} & -3 \\
\sqrt{2} & 2 & -\sqrt{2} \\
1 & \sqrt{1 / 2} & 0
\end{array}\right)
$$

where $U_{1}$ and $\ddot{U}_{2}$ are unitary matrices such that

$$
U_{1}^{H} A U_{1}=R_{1}=\left(\begin{array}{ccc}
1 & 1 & 4 \\
0 & 2 & 2 \\
0 & 0 & 3
\end{array}\right) \quad \text { and } \quad U_{2}^{H} A U_{2}=R_{2}=\left(\begin{array}{ccc}
2 & -1 & 3 \sqrt{2} \\
0 & 1 & \sqrt{2} \\
0 & 0 & 3
\end{array}\right) .
$$

The Schur decompositions in (18) show that $A$ has eigenvalues $\lambda(A)=\{1,2,3\}$ and that $A$ is nonnormal since $R_{1}$ and $R_{2}$ are not diagonal matrices. Moreover, the strictly upper triangular parts of the decompositions show that $\operatorname{dep}_{F}(A):=\|T\|_{F}=\sqrt{21}$.

The value of $\operatorname{dep}_{F}(A)$ is impractical to compute if $A$ is large and its eigenvalues are unknown. Lower bounds for $\operatorname{dep}_{F}(A)$ have been derived by Eberlein [3]

$$
\operatorname{dep}_{F}(A) \geq \frac{\left\|A^{H} A-A A^{H}\right\|_{F}}{\sqrt{6}\|A\|_{F}}
$$

and Loizou [7, Theorem 2]

$$
\operatorname{dep}_{F}(A) \geq \frac{\left\|A^{H} A-A A^{H}\right\|_{F}}{\beta^{1 / 2}+\left(\beta+\sqrt{2}\left\|A^{H} A-A A^{H}\right\|_{F}\right)^{1 / 2}}
$$

where

$$
\beta=\|A\|_{F}^{2}-\frac{1}{n}|\operatorname{trace}(A)|^{2} \text { and } A \neq 0 .
$$

The following upper bound has been derived by Henrici.

Theorem 2.5. [5, Theorem 1] For any $n \times n$ matrix $A$,

$$
\operatorname{dep}_{F}(A) \leq\left(\frac{n^{3}-n}{12}\right)^{1 / 4}\left(\left\|A^{H} A-A A^{H}\right\|_{F}\right)^{1 / 2}
$$


The bounds (19), (20) and (22) reduce to zero when $A$ is a normal matrix. Unfortunately, such bounds involve the matrix-matrix computation $A^{H} A-A A^{H}$, which generally requires $O\left(n^{3}\right)$ multiplications to obtain.

\section{A Sharp Upper Bound}

In deriving an upper bound for $\operatorname{dep}_{F}(A)$, a trivial bound comes from its definition:

$$
\operatorname{dep}_{F}(A)=\left(\|A\|_{F}^{2}-\|\Lambda\|_{F}^{2}\right)^{1 / 2} \leq\|A\|_{F}
$$

We can obtain better upper bounds for $\operatorname{dep}_{F}(A)$ by manipulating expressions that arise after splitting $A$ into its Hermitian part $M$ and skew-Hermitian part $N$.

Lernma 3.1. If $A=M-N$ has the Schur decomposition $U^{H} A U=\Lambda+T$, then

$$
\begin{aligned}
& \|M\|_{F}^{2}=\|\operatorname{Re}(\Lambda)\|_{F}^{2}+\frac{1}{2}\|T\|_{F}^{2}, \\
& \|N\|_{F}^{2}=\|\operatorname{Im}(\Lambda)\|_{F}^{2}+\frac{1}{2}\|T\|_{F}^{2} .
\end{aligned}
$$

Proof: The following chain of equalities follows directly from the definitions of the Frobenius norm, and the functions $\mathcal{H}(\cdot)$ and $\mathcal{S}(\cdot)$ :

$$
\begin{gathered}
\|M\|_{F}^{2}=\|\mathcal{H}(A)\|_{F}^{2}=\|\mathcal{H}(R)\|_{F}^{2}=\|\mathcal{H}(\Lambda)\|_{F}^{2}+\|\mathcal{H}(T)\|_{F}^{2}=\|\operatorname{Re}(\Lambda)\|_{F}^{2}+\frac{1}{2}\|T\|_{F}^{2}, \\
\|N\|_{F}^{2}=\|\mathcal{S}(A)\|_{F}^{2}=\|\mathcal{S}(R)\|_{F}^{2}=\|\mathcal{S}(\Lambda)\|_{F}^{2}+\|\mathcal{S}(T)\|_{F}^{2}=\|\operatorname{Im}(\Lambda)\|_{F}^{2}+\frac{1}{2}\|T\|_{F}^{2}
\end{gathered}
$$

These equalities can also be found in [8, pg. 495].

Equations (26) and (27) show that $\operatorname{dep}_{F}(A)$ can be defined in two different, but equivalent, ways:

$$
\operatorname{dep}_{F}(A)=\sqrt{2}\left(\|M\|_{F}^{2}-\|\operatorname{Re}(\Lambda)\|_{F}^{2}\right)^{1 / 2}
$$

or

$$
\operatorname{dep}_{F}(A)=\sqrt{2}\left(\|N\|_{F}^{2}-\|\operatorname{Im}(\Lambda)\|_{F}^{2}\right)^{1 / 2} .
$$

A simple upper bound for $\operatorname{dep}_{F}(A)$ follows directly from these equalities. 
Lemma 3.2. If $A=M-N$, where $M$ is the Hermitian part of $A$ and $N$ is the skew-Hermitian part of $A$, then

$$
\operatorname{dep}_{F}(A) \leq \sqrt{2} \min \left\{\|M\|_{F},\|N\|_{F}\right\}
$$

Proof: By breaking the equalities (28) and (29), we obtain

$$
\begin{aligned}
& \operatorname{dep}_{F}(A)=\sqrt{2}\left(\|M\|_{F}^{2}-\|\operatorname{Re}(\Lambda)\|_{F}^{2}\right)^{1 / 2} \leq \sqrt{2}\|M\|_{F} \\
& \operatorname{dep}_{F}(A)=\sqrt{2}\left(\|N\|_{F}^{2}-\|\operatorname{Im}(\Lambda)\|_{F}^{2}\right)^{1 / 2} \leq \sqrt{2}\|N\|_{F},
\end{aligned}
$$

and the upper bound

$$
\operatorname{dep}_{F}(A) \leq \sqrt{2} \min \left\{\|M\|_{F},\|N\|_{F}\right\}
$$

We now consider the use of the complex shift $\alpha I$ for improving the bound (30). As in Lemma 3.1, we can split $A-\alpha I$ into its Hermitian part $M-\operatorname{Re}(\alpha) I$ and skewHermitian part $N-\operatorname{Im}(\alpha) I$ and rearrange terms to obtain

$$
\operatorname{dep}_{F}(A)=\operatorname{dep}_{F}(A-\alpha I)=\sqrt{2}\left(\|M-\operatorname{Re}(\alpha) I\|_{F}^{2}-\|\operatorname{Re}(\Lambda)-\operatorname{Re}(\alpha) I\|_{F}^{2}\right)^{1 / 2}
$$

and

$$
\operatorname{dep}_{F}(A)=\operatorname{dep}_{F}(A-\alpha I)=\sqrt{2}\left(\|N-\operatorname{Im}(\alpha) I\|_{F}^{2}-\|\operatorname{Im}(\Lambda)-\operatorname{Im}(\alpha) I\|_{F}^{2}\right)^{1 / 2}
$$

A tighter bound can be obtained by minimizing the terms

$$
\|\operatorname{Re}(\Lambda)-\operatorname{Re}(\alpha) I\|_{F}^{2} \quad \text { and } \quad\|\operatorname{Im}(\Lambda)-\operatorname{Im}(\alpha) I\|_{F}^{2}
$$

before dropping them from equations (34) and (35), respectively. In particular,

$$
f_{1}(\operatorname{Re}(\alpha))=\|\operatorname{Re}(\Lambda)-\operatorname{Re}(\alpha) I\|_{F}^{2}=\sum\left(\operatorname{Re}\left(\lambda_{i}\right)-\operatorname{Re}(\alpha)\right)^{2}
$$

and

$$
f_{2}(\operatorname{Im}(\alpha))=\|\operatorname{Im}(\Lambda)-\operatorname{Im}(\alpha) I\|_{F}^{2}=\sum\left(\operatorname{Irn}\left(\lambda_{i}\right)-\operatorname{Im}(\alpha)\right)^{2}
$$


are quadratic functions that can be minimized using standard calculus techniques. By solving $f_{1}^{\prime}=0$ and $f_{2}^{\prime}=0$, we find that

$$
\operatorname{Re}(\alpha)=\frac{\sum \operatorname{Re}\left(\lambda_{i}\right)}{n} \text { and } \operatorname{Im}(\alpha)=\frac{\sum \operatorname{Im}\left(\lambda_{i}\right)}{n} .
$$

These values minimize equations $(37)$ and (38), respectively, since $f_{1}^{\prime \prime}$ and $f_{2}^{\prime \prime}$ are positive. Hence, both terms of equation (36) are minimized by choosing

$$
\alpha=\operatorname{Re}(\alpha)+\operatorname{Im}(\alpha)=\frac{\sum \operatorname{Re}\left(\lambda_{i}\right)}{n}+\frac{\sum \operatorname{Im}\left(\lambda_{i}\right)}{n}=\frac{\sum \lambda_{i}}{n}=\frac{\operatorname{trace}(A)}{n}
$$

Theorem 3.3. If $A=M-N$, where $M$ is the Hermitian part of $A$ and $N$ is the skew-Hermitian part of $A$, then

$$
\operatorname{dep}_{F}(A) \leq \sqrt{2} \min \left\{\|M-\operatorname{Re}(\alpha) I\|_{F},\|N-\operatorname{Im}(\alpha) I\|_{F}\right\}
$$

where the upper bound is minimized for

$$
\alpha=\frac{\operatorname{trace}(A)}{n} .
$$

Moreover, the bound is sharp (i.e., equality holds) if and only if the eigenvalues of $A$ are horizontally or vertically aligned in the complex plane.

Proof: The upper bound is obtained from equations (34) and (35) by dropping the terms $\|\operatorname{Re}(\Lambda)-\operatorname{Re}(\alpha) I\|_{F}^{2}$ and $\|\operatorname{Im}(\Lambda)-\operatorname{Im}(\alpha) I\|_{F}^{2}$, respectively. The bound is sharp iff

$$
\begin{aligned}
& \|\operatorname{Re}(\Lambda)-\operatorname{Re}(\alpha) I\|_{F}^{2}=0 \text { or } \\
& \|\operatorname{Im}(\Lambda)-\operatorname{Im}(\alpha) I\|_{F}^{2}=0 .
\end{aligned}
$$

The first condition (43) says that the real parts of the eigenvalues of $A$ are constant (i.e., vertically aligned). The second condition (44) says that the imaginary parts of the eigenvalues of $A$ are constant (i.e., horizontally aligned).

Equations (34) and (35) also show that

$$
\operatorname{dep}_{F}(A) \approx \sqrt{2} \min \left\{\|M-\operatorname{Re}(\alpha) I\|_{F},\|N-\operatorname{Im}(\alpha) I\|_{F}\right\}
$$

iff

$$
\begin{gathered}
\|\operatorname{Re}(\Lambda)-\operatorname{Re}(\alpha) I\|_{F}^{2} \ll\|M-\operatorname{Re}(\alpha) I\|_{F}^{2} \quad \text { or } \\
\|\operatorname{Im}(\Lambda)-\operatorname{Im}(\alpha) I\|_{F}^{2} \ll\|N-\operatorname{Im}(\alpha) I\|_{F}^{2}
\end{gathered}
$$


Hence, the new bound (41) is a good approximation when the eigenvalues of $A$ are relatively close to being horizontally or vertically aligned: otherwise, the bound is weat.

Example 2. Let's compare the Henrici bound (22) and the new bound (11) for the matrix $A$ in Example 1, in which $\operatorname{dep}_{F}(A)=\sqrt{21}$. Using the intermediate quantities

$$
\left\|A^{H} A-A A^{H}\right\|_{F}=\sqrt{996}, \alpha=2,\|M-2 I\|_{F}=\sqrt{25 / 2} \text { and }\|N\|_{F}=\sqrt{21 / 2}
$$

the Henrici bound gives

$$
\operatorname{dep}_{F}(A) \leq(2)^{1 / 4}(\sqrt{996})^{1 / 2}=(\sqrt{1992})^{1 / 2} \approx \sqrt{44.6318}
$$

and the new bound gives

$$
\operatorname{dep}_{F}(A) \leq \sqrt{2} \min \{\sqrt{25 / 2}, \sqrt{21 / 2}\}=\sqrt{21}
$$

In this example, the new bound is tighter than the Henrici bound. The new bound is sharp since the eigenvalues of $A$ are strictly real. ${ }^{1}$

Numerous examples can be contrived for which the new bound is tighter than the Henrici bound or vice versa. In general, the new bound is preferable since the Henrici bound is an $O\left(n^{3}\right)$ computation and the new bound is an $O\left(n^{2}\right)$ computation. It is sometimes possible to further improve the new bound by rotating $A-\alpha l$. For complex matrices, the eigenvalues of $A-\alpha I$ can be arbitrarily distributed and the best rotation $\theta$ can not be determined a priori. For real matrices, the eigenvalues of $A-\alpha I$ occur in complex-conjugate pairs and the new bound is minimized for $\theta \in\left\{0, \frac{\pi}{2}, \pi, \frac{3 \pi}{2}\right\}$. Note that the implicit use of $\theta=0$ has the advantage that the new bound reduces to zero for Hermitian and skew-Hermitian matrices. Unfortunately, for normal matrices whose eigenvalues are not horizontally or vertically aligned, the new bound does not reduce to zero.

In contrast to the improvements for the new bound, the Henrici bound is unaffected by complex shifts and rotations of $A$. This is so because, for complex shifts of $A$,

$$
\begin{aligned}
& \left.\|(A-\alpha I)^{H}(A-\alpha I)-(A-\alpha I)(A-\alpha I)^{H}\right) \|_{F} \\
= & \left\|\left(A^{H} A-\alpha^{H} A-\alpha A^{H}+\alpha^{H} \alpha I\right)-\left(A A^{H}-\alpha^{H} A-\alpha A^{H}+\alpha \alpha^{H} I\right)\right\|_{F} \\
= & \left\|A^{H} A-A A^{H}\right\|_{F} .
\end{aligned}
$$

The same simplification (51)-(53) also occurs for the more general case of $e^{-i \theta}(A-\alpha I)$.

\footnotetext{
${ }^{1}$ For completeness, the Eberlein (19) and Loizou (20) lower bounds are 2.1778 and 2.4240, respectively.
} 


\section{Numerical Results}

Table 1 compares the upper bounds (22) and (41) for the nonnormal test matrices given by Eberlein in [2]. Loizou in [7] used this set of matrices to compare the two lower bound formulas (19) and (20). Table 1 shows the new upper bound is tighter than the Henrici upper bound for all 17 examples. Moreover, the new bound is sharp for test matrix $4,6,7,9,11$ and 17 .

Table 2 compares the upper bounds for some of the $32 \times 32$ nonnormal test matrices studied by Trefethen in [9]. In each case, the new upper bound is tighter than the Henrici upper bound. Moreover, the new bound is sharp for the mairices entitled: Jordan block, Limaçon, Wilkinson, Frank, Kahan, Demmel, Lenierink-Spijker, Companion, and Gauss-Seidel. Such good results are predicted by Theorem 3.3 since the aforementioned matrices have strictly rea' igenvalues and the other matricns (Grcar, Chebyshev spectral) have eigenvalues that are almost vertically aligned.

\begin{tabular}{|c||c|c|c||c|c|}
\hline \multirow{2}{*}{ Test matrix } & Henrici bound & new bound & $\operatorname{dep}_{F}(A)$ & \multicolumn{2}{|c|}{ ratio } \\
\hline 1 & 1.411 & $(41)$ & $(12)$ & $(22) /(12)$ & $(41) /(12)$ \\
\hline 2 & 0.141 & 1.005 & 0.99 & 1.43 & 1.02 \\
\hline 3 & 7.416 & 0.100 & 0.100 & 1.41 & 1.00 \\
\hline 4 & 5.214 & 1.610 & 2.449 & 3.03 & 1.40 \\
\hline 5 & 93.325 & 40.623 & 33.610 & 3.24 & 1. \\
\hline 6 & 1.778 & 1.732 & 1.732 & 2.82 & 1.23 \\
\hline 7 & 3.310 & 2.828 & 2.828 & 1.03 & 1. \\
\hline 8 & 21.644 & 10.955 & 10.895 & 1.99 & 1. \\
\hline 9 & 15.477 & 10.010 & 10.010 & 1.55 & 1. \\
\hline 10 & 95.933 & 46.033 & 45.924 & 2.09 & 1.00 \\
\hline 11 & 128.24 & 34.785 & 34.785 & 3.69 & 1. \\
\hline 12 & $1.584 \mathrm{e}+3$ & 361.994 & 357.659 & 4.43 & 1.01 \\
\hline 13 & $7.524 \mathrm{e}+3$ & 924.661 & 917.796 & 8.20 & 1.01 \\
\hline 14 & 86.759 & 75.974 & 58.481 & 1.48 & 1.30 \\
\hline 15 & 250.249 & 188.149 & 94.184 & 2.66 & 2.00 \\
\hline 16 & 95.933 & 46.033 & 45.924 & 2.09 & 1.00 \\
\hline 17 & 2.115 & 1.414 & 1.414 & 1.50 & 1. \\
\hline
\end{tabular}

Table 1: Departure from normality results for Eberlein [2] nonnormal test matrices. 


\begin{tabular}{|c|c|c|c|c|c|}
\hline & Henrici hound & new hound & $\operatorname{deg}_{F}(A)$ & \multicolumn{2}{|c|}{ ratio } \\
\hline Test matrix & $(22)$ & $(.11)$ & $(12)$ & $(22) /(12)$ & $(41) /(12)$ \\
\hline Jordan block & 8.594 & 5.568 & 5.568 & 1.54 & 1 \\
\hline Limaçon & 13.980 & 7.810 & 7.810 & 1.79 & 1. \\
\hline Grcar & 18.398 & 7.681 & 6.007 & 3.06 & 1.28 \\
\hline Tilkinson & 8.659 & 5.568 & 5.568 & $\overline{1.56}$ & 1. \\
\hline Frank & $1.821 e+3$ & $2.772 e+2$ & $2.772 \mathrm{e}+2$ & 6.57 & 1. \\
\hline Kahan & 38.092 & 4.982 & 4.982 & 7.65 & 1. \\
\hline Demmel & $1.236 \mathrm{e}+9$ & $1.438 \mathrm{e}+8$ & $1.438 \mathrm{e}+8$ & 8.59 & 1. \\
\hline Lenferink-Spijker & $.384 e+2$ & $1.067 \mathrm{e}+2$ & $1.067 \mathrm{e}+2$ & 2.23 & 1. \\
\hline Companion & $5.281 e+5$ & $6.14 \overline{j e}+4$ & $6.145 e+4$ & 8.59 & 1. \\
\hline Gauss-Seidel & 4.555 & 2.149 & 2.149 & 2.12 & 1. \\
\hline Chebysher spectral & 4.392 & 0.572 & 0.570 & 7.71 & 1.00 \\
\hline
\end{tabular}

Table 2: Departure from normality results for Trefethen [9] nonnormal test matrices.

\section{Acknowledgements}

I wish w thank Steve Ashby. Linda Petzold and Paul Saylor for the many suggestions and encouragements that sustained this investigation of bounding the departure from normality of a matrix. I also thank Ed D Azevedo and Faisal Sajed for many helpful discussions and for helping to strengthen the new bound. Finally, I am grateful to June Donato. Gene Golub and Paul Saylor for the valuable comments that have improved the presentation of this paper. 


\section{References}

[1] J. Descloux. Bounds for the spectral norm of functions of matrices. Numer. Math., 5:185-190, 1963.

[2] P. J. Eberlein. A Jacobi-like method for the automatic computation of eigenvalues and eigenvectors of an arbitrary matrix. SIAM J., 10:74-88, 1962.

[3] P. J. Eberlein. On measures of nun-normality for matrices. Amer. Math. Soc. Monthly, 72:995-996, 1965.

[4] G.H. Golub and C. Van Loan. Matrix Computations. The Johns Hopkins University Press, Baltimore, Maryland, 1989.

[5] P. Henrici. Bounds for iterates, inverses, spectral variation and fields of values of non-normal matrices. Numer. Math., 4:24-40, 1962.

[6] R.A. Horn and C.R. Johnson. Matrix Analysis. Cambridge University Press, Cambridge, 1990 .

[7] G. Loizou. Nonnormality and Jordan condition numbers of matrices. J. Assoc. Comp. Mach., 16(4):580-584, 1969.

[8] I. Schur. On the characteristic roots of a linear substitution with an application to the theory of integral equations. Math. Ann., 66:488-510 (German), 1909.

[9] L.N. Trefethen. Pseudospectra of matrices. In D.F. Griffiths and G.A. Watson, Numerical Analysis 1991, pages 234-266. Longman, 1991. 
ORNL/TM-12426

\section{INTERNAL DISTRIBUTION}

1. B. R. Appleton

2-3. T. S. Darland

4. E. F. D'Azevedo

5. J. M. Donato

6. J. J. Dongarra

7. J. B. Drake

8. G. A. Geist

9. L. J. Gray

10-14. S. L. Lee

15. M. R. Leuze

16. N. M. Nachtigal

17. C. E. Oliver
18. P. M. Papadopoulos

19. B. W. Peyton

20-24. S. A. Raby

25. C. H. Romine

26. B. D. Semeraro

27-31. R. F. Sincovec

32-36. R. C. Ward

37. Central Research Library

38. ORNL Patent Office

39. K-25 Appl Tech Library

40. Y-12 Technical Library

41. Lab Records Dept - RC

42-43. Laboratory Records Dept

\section{EXTERNAL DISTRIBUTION}

44. Steve Ashby, Lawrence Livermore National Laboratory, P.O. Box 808, L-316, Livermore, CA 94551

45. Zhaojun Bai, Department of Mathematics, University of Kentucky, Lexington, KY 40506

46. Jesse Barlow, Department of Computer Science and Engineering, 220 Pond Laboratory, The Pennsylvania State University, University Park, PA 16802-6106

47. Michael Berry, Department of Computer Science, 114 Ayres Hall, University of Tennessee, Knoxville, TN 37996-1301

48. Randall Bramley, Department of Computer Science, 215 Lindley Hall, Indiana University, Bloomington, IN 47405

49. Roger W. Brockett, Pierce Hall 29 Oxford Street Harvard University Cambridge, MA 02138

50. Peter Brown, Lawrence Livermore National Laboratory, P.O. Box 808, L-316, Livermore, CA 94551

51. Ralph Byers, 405 Snow Hall, Department of Mathematics, University of Kansas, Lawrence, KS 66045

52. Tony Chan, Department of Mathematics, UCLA, 405 Hilgard Avenue, Los Angeles, CA 90024

53. Françoise Chatelin, IBM CEMAP, 68-76 Quai de la Rapée, 75592 Paris Cedex 12, France

54. Alexandre Chorin, Mathematics Department, Lawrence Berkeley Laboratory, Berkeley, CA 94720 
55. Paul Concus, Lawrence Berkeley Laboratory, 50A-2129, University of California, Berkeley, CA 94720

56. James Corones, Ames Laboratory, Iowa State University, Ames, 1 A 50011

57. James Demmel, Computer Science Division, Department of EECS, 513 Evans IIall, University of California at Berkeley, Rerkeley, CA 94720

58. Milo Dorr, Lawrence Livermore National Laboratory, P.O. Box 808, L-316, Livermore, CA 94551

59. Augustin Dubrulle, Hewlett-Packard Labs., 1501 Page Mill Rd., 3U-7, Palo Alto. CA 94304

60. Donald J. Dudziak, Department of Nuclear Engineering, 110B Burlington Engineering Labs, North Carolina State University, Raleigh, NC 27695-7909

61. Patricia Eberlein, Department of Computer Science, State University of New York, 226 Bell Hall, Buffalo, NY 14260

62. Michael Eiermann, Universtät Karlsruhe, Institut für Praktische Mathematik, Englerstr. 2, D-7500 Karlsruhe, Germany

63. Victor Eijkhout, Department of Computer Science, 104 Ayres Hall, University of Tennessee, Knoxville, TN 37996-1301

64. Stanley C. Eisenstat, Department of Computer Science, Yale University, P.O. Box 2158, Yale Station, New Haveı, CT 06520

65. Howard C. Elman, Computer Science Department, University of Maryland, College Park, MD 20742

66. Richard E. Ewing, Department of Mathematics, Texas A\&M University, College Station, TX 77843

67. Rob Falgout, Lawrence Livermore National Laboratory, P.O. Box 808, L-419, Livermore, CA 94551

68. Bernd Fischer, Institut of Applied Mathematics, University of Hamburg, Bundesstrasse 55, 2000 Hamburg 13, Germany

69. Valérie Frayssé, CERFACS, 31057 Toulouse Cedex, France

70. Roland Freund, AT\&'T Bell Labs., Room 2C-420, 600 Mountain Avenue, Murray Hill, NJ 07974-0636

71. Robert Funderlic, Computer Science Department, Box 8206, 228 Withers Hall, North Carolina State University, Raleigh. NC 27695-8206

72. Stratis Gallopoulos, Department of Computer Science, University of Illinois at lirbana-Champaign, 465 CSRL, 1308 West Main St., Urbana, IL 61801

73. Walter Gander, Institut für Wissenschaftliches Rechnen, ETH-Zentrum, CH-8092 Zurich, Switzerland

74. C. William Gear, NEC Research Institute, 4 Independence Way. Princeton, NJ 08540-6621

75. James Glimm, Department of Mathematics, State University of New York, Stony Brook, NY 11794 
76. Gene H. Golub, Department of Computer Science, Stanford University, Stanford, CA 94305

77. Joe Grcar, Dept. 8745, Sandia National Laboratories, Livermore, CA 94551-0969

78. Anne Greenbaum, Courant Institute of Mathematical Sciences, New York University, 251 Mercer St., New York, NY 10012-1185

79. Martin Gutknecht, IPS Supercomputing, ETH Zurich, ETH-Zentrum, CH-8092 Zurich, Switzerland

80. Michael T. Heath, National Center for Supercomputing Applications, 4157 Beckman Institute, University of Illinois, 405 North Mathews Avenue, Urbana, IL 61801-2300

81. Bruce Hendrickson, Sandia National Laboratories, Division 1422, Albuquerque, NM 87185

82. Nick Higham, Department of Mathematics, University of Manchester, 90 Half Edge Lane, Eccles, Manchester, M13 9PL, United Kingdom

83. Alan Hindmarsh, Lawrence Livermore National Laboratory, P.O. Box 808, L-316, Livermore, CA 94551

84. Michael J. Holst, California Institute of Technology, Applied Mathematics 217-50, Pasadena, CA 91125

85. Fred Howes, Office of Scientific Computing, ER-7, Applied Mathematical Sciences, Office of Energy Research, Department of Energy, Washington, DC 20585

86. James M. Hyman, Los Alamos National Laboratory, MS B284, Group 'T-7, Los Alamos, NM 87545

87. Khakim Ikramov, Computer Science Deparment, University of Waterloo, Waterloo, Ontario, Canada N2L 3G1

88. Ilse Ipsen, Department of Computer Science, Yale University, P.O. Box 2158, Yale Station, New Haven, CT 06520

89. Erxiong Jiang, Department of Mathematics, Fudan University, Shanghai 200433, People's Republic of China

90. Gary Johnson, Office of Scientific Computing, ER-7, Applied Mathematical Sciences, Office of Energy Research, Department of Energy, Washington, DC 20585

91. Bo Kågström, Institute of Information Processing, University of Umeå, S-901 87 Umeå, Sweden

92. Malvyn H. Kalos, Cornell Theory Center, Engineering and Theory Center Bldg., Cornell University, Ithaca, NY 14853-3901

93. Hans Kaper, Mathematics and Computer Science Division, Argonne National Laboratory, 9700 South Cass Avenue, Bldg. 221, Argonne, IL. 60439

94. David Kincaid, Center for Numerical Analysis, RLM Hall 13.150 University of Texas at Austin, Austin, TX 78713-8510

95. Alan Laub, ECE Department, University of California, Santa Barbara, Santa Barbara, CA 93106-9560

96. Peter D. Lax, Courant Institute of Mathematical Sciences, New York University, 251 Mercer Street, New York, NY 10012 
97. James E. Leiss, Rt. 2, Box 142C, Broadway, VA 22815

98. Steven J. Leon, Department of Mathematics, University of Massachusetts, Old Wes'.port Road, North Dartmouth, MA 02747

99. Tom Manteuffel, Program in Applied Mathematics, Campus Box 526, University of Colorado at Boulder, Boulder, CO 80309-0526

100. Juan Meza, Sandia National Laworatories. Division 8211, P.O. Box 969, Livermore, CA $94551-0969$

101. Cleve Moler, The MathWorks, 24 Prime Park Way, Natick, MA 01760

102. Neville Moray, Department of Mechanical and Industrial Engineering, University of Illinois, 1206 West Green Street, Urbana, IL 61801

103. David Nelson, Director of Office of Scientific Computing Staff, ER-7, Applied Mathematical Sciences, Office of Energy Research, Department of Energy, Wishington, DC 20585

104. Dianne P. O'Leary, Computer Science Department, University of Maryland, College Park, MD 20742

105. Beth Ong, University of California, San Diego, Department of Mathematics, 9500 Gilman Drive, La Jolla, CA 92093-0112

106. James M. Ortega, Department of Applied Mathematics, Thornton Hall, University of Virginia, Charlottesville, VA 22901

107. Chris Paige, McGill University, School of Computer Science, M-Connell Engineering Building, 3480 University Street, Montreal, Quebec, Canada H3A 2A7

108. Beresford Parlett, Mathematics Department, University of California, Berkeley, Berkeley, CA 94720

109. Linda Petzold, Department of Computer Science, 4-192 EE/CS Building, 200 Union Street SE, University of Minnesota, Minneapolis, MN 55455

110. Satish Reddy, Department of Mathematics, Oregon State University, 344 Kidder Hall, Corvallis, OR 97331

111. Lothar Reichel, Department of Mathematics and Computer Science, Kent State University, Kent, OH 44242

112. Axel Ruhe, Department of Computer Science, Chalmers, S-41296 Göteborg, Sweden

113. Youcef Saad, Department of Computer Science, 4-192 EE/CS Building, 200 Union Street SE, University of Minnesota, Minneapolis, MN 55455-0154

114. Faisal Saied, Department of Computer Science, 1304 W. Springfield Ave, University of Illinois at Urbana-Champaign, Urbana, IL 61801

115. Ahmed H. Sameh, Department of Computer Science, 4-192 EE/CS Building, 200 Union Street SE, University of Minnesota, Minneapolis, MN 55455

116. Paul Saylor, Department of Computer Science, 1304 W. Springfield Ave, University of Illinois at Urbana-Champaign, Urbana, IL 61801

117. Martin Schultz, Department of Computer Science, Yale University, P.O. Box 2158 Yale Station, New Haven, CT 06520 
118. John Shadid, Sandia National Laboratories, Parallel Computer Science Division, Division 1421, Albuquerque, NM 87185

119. Bob Skeel, Department of Compute' Science, 1304 W. Springfield Ave, University of Illinois at Urbana-Champaign, Urbana, IL 61801

120. Gerhard Starke, Computational Math Group, University of Colorado at Denver, Diver, CO $802(04$

121. G.W. Stewart, Conipiater Science Department, University of Maryland, College Park, MD 20742

122. Zdenek Strakos, Institute of Computer Science, ICS AS of the Czech Republic, Por vod. vezi 2, 18207 Praha 8, Czech Republic

123. Gilbert Strang, Massachusetts Institute of Technology, Room 2-240, Cambridge, MA 02319-4557

124. Ji-guang Sun, Institute of Information Processing, University of Umeå, S-901 87 Umeå, Sweden

125. Xiaobai Sun, Argonne National Laboratory, Bldg. 221, MCS Division, 9700 South Cass Ave., Argonne, IL 60439

126. Toshio Suzuki, Department of Mathematics, Yamanashi University, Kofu 400, Japan

127. Charles Tong, Sandia National Laboratories, Department 8117, Livermore, CA 94551-0969

128. Anne Trefethen, Cornell University, Dept. of Computer Science, Ithaca, NY 14853

129. Lloyd N. Trefethen, Cornell University, Dept. of Computer Science, Ithaca, NY 14853

130. Paul Van Dooren, Coordinated Science Laboratory, University of Illinois at UrbanaChampaign, 1308 W. Main St., Urbana, IL 61801

131. Charles Van Loan, Computer Science Department, Cornell University, lthaca, NY 14853

132. James Varah, CICSR Office, CEME 2053, University of British Columbia, Vancouver BC, Canada

133. Richard S. Varga, Department of Mathematics, Kent State University, Kent, $\mathrm{OH}$ 44242

134. Daniel Warner, Department of Mathematical Sciences, O-104 Martin Hall, Clemson University, Clemson, SC 29631

135. Andy Wathen, School of Mathematics, University Walk, Bristol University, Bristol BS8 1TW, United Kingdom

136. Mary F. Wheeler, Rice University, Department of Mathematics, P.O. Box 1892 , Houston, TX 77251

137. David Young, University of Texas, Center for Numerical Analysis, RLM 13.150, Austin, TX 78731

138. Hongyuan Zha, Department of Computer Science and Engineering, 220 Pond Laboratory, The Pennsylvania State University, University Park, PA 16802-6106 
139. Office of Assistant Manager for Energy Research and Development, Department of Energy, Oak Ridge Operations Office, P.O. Box 2001 Oak Ridge. TN 37831-8600

140-141. Office of Scientific \& Technical Information, P.O. Box 62. Oak Ridge, TN 37831 

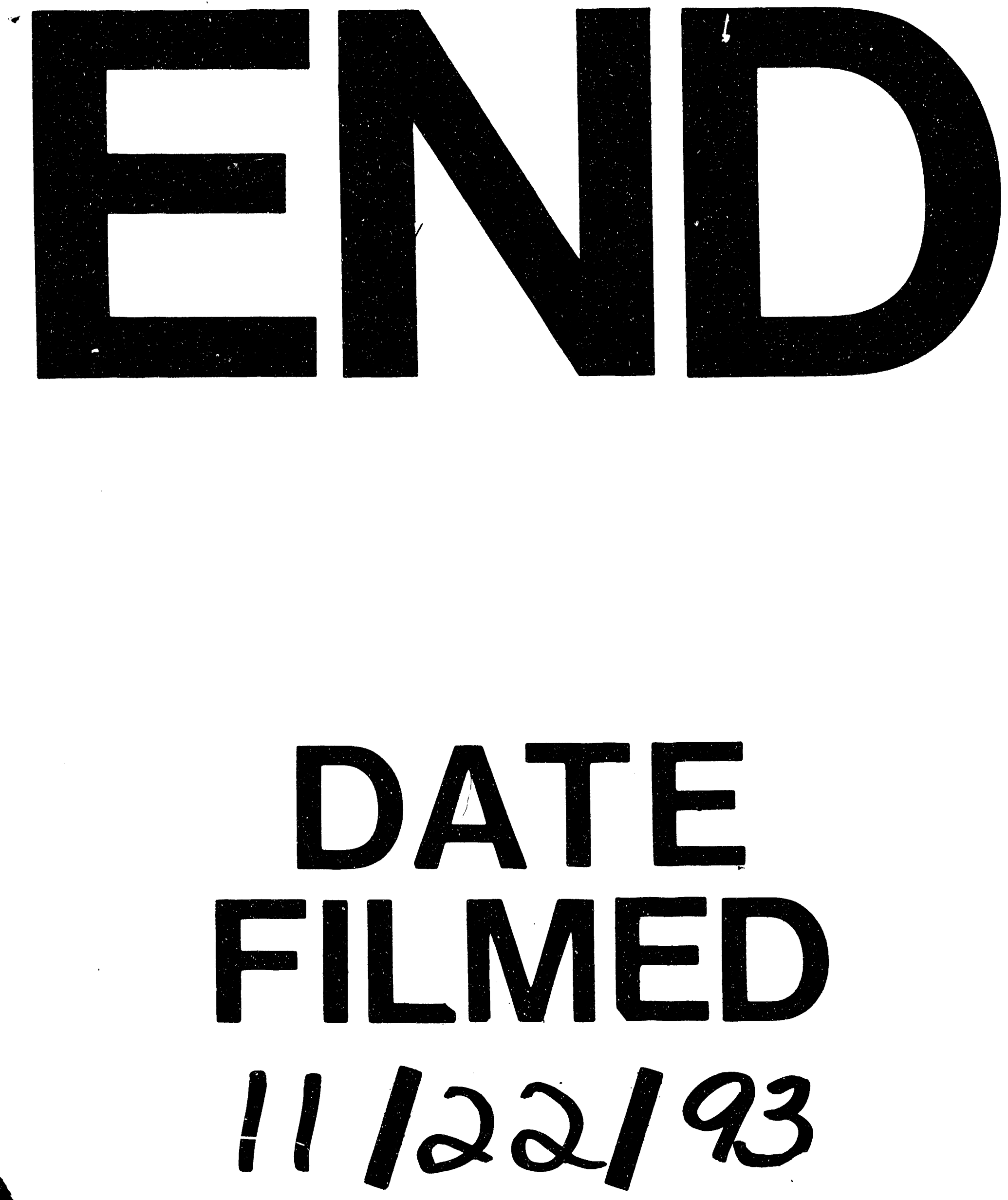
